mit geringer langwelliger Tilgbarkeit verbundenen Erregung durch Kathodenstrahlen naheliegen.

e) $\mathrm{Zu}$ den Nebenvorgängen, welche jeder Deutungsversuch der Erscheinungen wird mit berücksichtigen müssen, gehört bei der Photoluminescenz die Veränderung der erregenden Ab-

${ }_{14}$ Handb. d. Exp.-Physik, S. 692 [1928]; A. H o c h, Ann. Physik 38, 486 [1940]. sorption ${ }^{14}$ durch die Tilgung, deren gesonderte Messung noch aussteht und daher anzustreben ist. Wie weit bei der Radioluminescenz oder der Elektronenanregung die im Grundmaterial allgemein erzeugte Leitfähigkeit ${ }^{15}$ eine Rolle spielt, entzieht sich ebenfalls unserer Kenntnis.

15 A. B e ck e r, Ann. Physik 12, 124 [1903]; 13, 394 [1904]; R. L a p p e, Ann. Physik 39, 604 [1941].

\title{
Der geometrische Ort für die mittlere Geschwindigkeit bei turbulenter Strömung in glatten und rauhen Rohren
}

\author{
Von Wilhelm Aichelen*
}

\author{
(Z. Naturforschg. 2 a, 108-110 [1947]; eingegangen am 21. Dezember 1946)
}

\begin{abstract}
Es wird gezeigt, daß bei ausgebildeter turbulenter Strömung im glatten und rauhen Rohr beim Radius $r=0,762 r_{0}$ bei allen $R$ e $y$ old schen Zahlen die mittlere Geschwindigkeit herrscht $\left(r_{0}=\right.$ Rohrradius). Ein auf dieser Tatsache begründetes neues Mengenmeßgerät wird vorgeschlagen.
\end{abstract}

$\mathrm{D}$ ie turbulente Strömung in glatten Rohren spielt in der Technik eine bedeutende Rolle. Sie ist deshalb der Gegenstand zahlreicher ausführlicher Untersuchungen gewesen, die u. a. von Stanton ${ }^{1}$, Pannell, Bazin, Schiller ${ }^{2}$ und Herrmann ${ }^{3}$ durchgeführt wurden. Die letzten und genauesten Messungen auf diesem Gebiet stammen wohl von $\mathrm{Nikur}$ a d s e ${ }^{4}$, der seine Versuche mit Wasser und Rohren verschiedenster Durchmesser durchführte und sie bis zu den höchsten Reynoldsschen Zahlen ausgedehnt hat. Alle Experimentatoren haben neben dem Druckabfall auch eingehend die Geschwindigkeitsverteilung untersucht und das Verhältnis der mittleren Geschwindigkeit zur maximalen Geschwindigkeit in Abhängigkeit der R e y n o lds schen Zahl dargestellt. Jedoch ist nach unserem Wissen der Frage noch keine Beachtung geschenkt worden, bei welchem Radiusverhältnis die mittlere Geschwindigkeit auftritt, und ob sich dieses

* Jetzt Neunkirchen (Saar), Wellesweilerstr. 78. Die Untersuchung wurde im Maschinenlaboratorium der Technischen Hochschule Danzig ausgeführt.

1 T. E. Stanton, The mechanicle Viscosity of Fluids. Proc. Roy. Soc. [London] Ser. A, S. 366 [1911].

2 Schiller, Rohrwiderstand bei hohen Rey n o lds schen Zahlen. Vorträge aus dem Gebiet der Aerodynamik und verwandter Gebiete, Aachen [1929]. Herausgeg. von A. Gilles, L. Ho p f u. Th. v. K á r má n, Berlin 1930.
Radiusverhältnis in Abhängigkeit von der R e y noldsschen Zahl gesetzmäßig erfassen läßt. Einen Beitrag zu dieser Frage soll folgende Arbeit leisten.

\section{Versuche mit Luft}

Die Versuche wurden in einem kreisrunden, glatten Rohr mit Luft durchgeführt. Die verschiedenen Luftgeschwindigkeiten wurden durch Regelung der Drehzahl eines Hochdruckventilators eingestellt. An das Druckrohr des Ventilators wurde ein Behälter angeschlossen, dessen Deckelfläche aus einer Gummimembrane bestand. Dieser Ausgleichsbehälter wurde mit dem Meßrohr durch einen Gummiflansch verbunden, so daß die durch den Ventilator verursachten Erschütterungen nicht auf die Meßstelle übertragen werden konnten. Zur Erzielung einer gut ausgeprägten turbulenten Strömung im Meßquerschnitt wurde als Anlaufstrecke ein $6450 \mathrm{~mm}$ langes Eisenrohr von $82 \mathrm{~mm}$ Innendurchmesser gewählt. Der Einlauf in das Eisenrohr wurde scharfkantig ausgeführt. Um einen möglichst gleichmäßigen Ubbergang in das eigentliche

3 R. H e r r m a n n, Experimentelle Untersuchung zum Widerstandsgesetz des Kreisrohres bei hohen R e y n o ld s schen Zahlen und großen Anlauflängen. Diss. Akademische Verlagsgesellschaft m.b.H., Leipzig 1930.

4 J. Nikuradse, Gesetzmöglichkeiten der turbulenten Strömungen in glạtten Rohren, Forschungshefte 356 [1932]; Widerstandsgesetz und Geschwindigkeitsverteilung von turbulenten Wasserströmungen in glatten und rauhen Rohren, Verh.3. Intern. Kongr. f. techn. Mech., S. 239. Strömungsgesetze in rauhen Rohren, Forschungshefte 361 [1933]. 
Versuchsrohr zu bekommen, wurde das Verbindungsstück auf einer Länge von $500 \mathrm{~mm}$ konisch auf den Innendurchmesser des Versuchsrohres verjünt. Das innen glatte Versuchsrohr aus gezogenem Messing mit $65,3 \mathrm{~mm}$ Innendurchmesser und $2874 \mathrm{~mm}$ Länge war auf einem Holzbock fest gelagert. Der Querschnitt wurde mit einem Staurohr von $1 \mathrm{~mm}$ Innendurchmesser abgetastet und die mittlere Geschwindigkeit durch graphische Integration der Gleichung

$$
w_{m}=\frac{2}{r_{0}^{2}} \int_{0}^{r_{0}} w r d r
$$

bestimmt; hierin bedeuten: $w_{m}$ mittlere Geschwindigkeit, $w$ jeweilige Geschwindigkeit, $r$ jeweiliger Abstand von der Mitte des Rohres, $r_{0}$ Rohrradius.

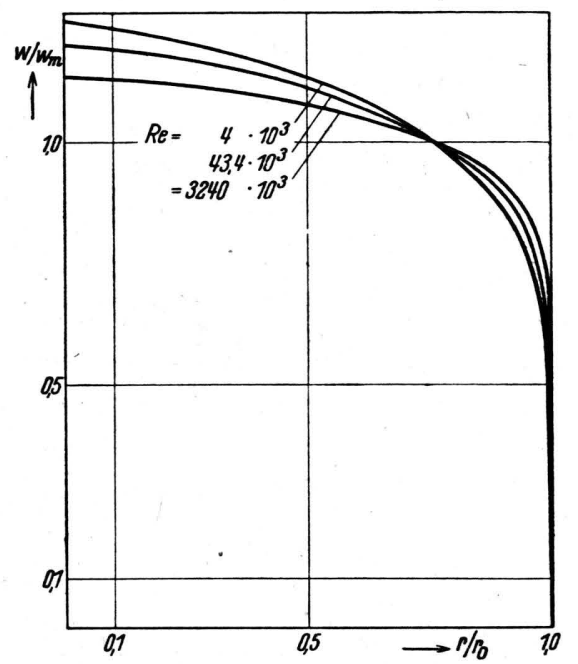

Abb. 1. $w / w_{m}$ in Abhängigkeit von $r / r_{0}$ nach $\mathrm{Ni} \mathrm{k} \mathrm{u} \mathrm{r} \mathrm{a} \mathrm{d} \mathrm{e.}$

Die aufgenommenen Strömungsprofile unterscheiden sich nur unwesentlich von den Meßergebnissen der angegebenen Autoren. $\mathrm{Niku}$ r adse mißt unmittelbar an der Rohrinnenwand eine endliche Geschwindigkeit. Diesen Meßpunkt verwerfen wir, da er nur in der Art der Messung begründet liegt. Wie $\mathrm{Nikuradse}$ an anderer Stelle angibt, herrscht natürlich unmittelbar an der Rohrinnenwand die Geschwindigkeit null.

Die durch graphische Auswertung gefundene mittlere Geschwindigkeit wurde nun in die aufgenommenen Strömungsprofile eingetragen. Es zeigt sich dabei die überraschende Tatsache, daß bei den verschiedensten R e y nolds schen Zahlen der mittlere dynamische Druck und damit die mittlere Geschwindigkeit immer beim selben Radius gemessen wurde (Abb.1).
Um dieses Ergebnis zu unterbauen, wurden nun noch Meßergebnisse anderer Autoren herangezogen und ausgewertet. $\mathrm{Nikurat} \mathrm{s} \mathrm{e}^{4}$ hat Versuche bei den verschiedensten $R$ ey noldsschen Zahlen im Bereich von $4 \cdot 10^{3}$ bis $3,24 \cdot 10^{6}$ mit Wasser und Stanto $\mathrm{n}^{\mathbf{1}}$ bei drei verschiedenen Rey noldsschen Zahlen mit Luft durchgeführt. Es wurde der Wert des Radiusverhältnisses an der Stelle $w / w_{m}=1$ ermittelt und über der dazugehörigen $\mathrm{R}$ e y noldsschen Zahl aufgetragen (Abb.2). Aus Abb. 2 ersieht man, daß die Punkte, die aus den Arbeiten dreier verschiedener Autoren entnommen sind, mit großer Genauigkeit auf einer Parallelen zur Abszisse liegen.

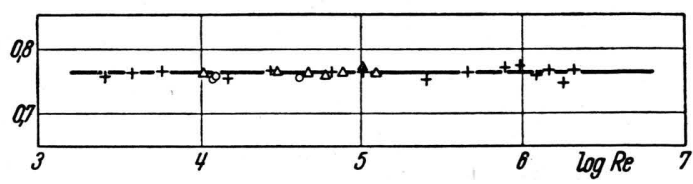

Abb. 2. $r / r_{0}$ an der Stelle $w / w_{m}=1$ in Abhängigkeit von der $\mathrm{Reynoldsschen} \mathrm{Zahl} \mathrm{für} \mathrm{glatte} \mathrm{Rohre.}$ $(+\mathrm{Nikurads}$ e [Wasser], $\triangle \mathrm{Eigene}$ Messungen [Luft], O Stant on [Luft].)

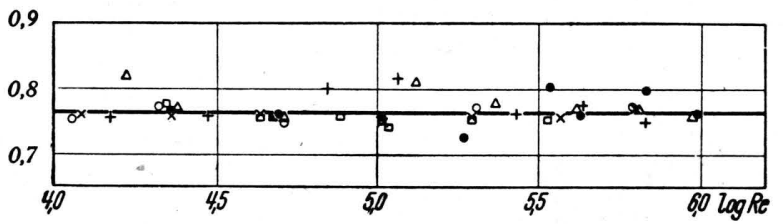

Abb. 3. $r / r_{0}$ an der Stelle $w / w_{m}=1$ in Abhängigkeit von der Rey noldsschen Zahl für rauhe Rohre. (Rauhigkeit $r_{0} / k: \bullet 507,0252, \Delta 126,+60, \times 30,6$, 口 15 [nach Nikuradse]; [ [Stanton].)

Die Ordinate hat den Wert $r / r_{0}=0,762$.

Zur Prüfung der Verhältnisse im rauhen Rohr wurden wieder Messungen von Stanto $\mathrm{n}^{1}$ und $\mathrm{Nikuradse}^{4}$ herangezogen und ausgewertet. Letzterer hat bei exakt definierter Rauhigkeit Versuche durchgeführt. Dabei wurde die Rauhigkeit so erzeugt, daß Sandkörner gleicher, bestimmter Korngröße in das zu untersuchende Rohr eingeklebt wurden und dann aus ihrem Durchmesser und dem Durchmesser des Rohres der Grad der Rauhigkeit bestimmt wurde. Die Rauhigkeit wurde in dem Bereich von $r_{0} / k=507$ bis $r_{0} / k=15$ verändert, wobei $k$ die mittlere Erhebung der Rauhigkeit bedeutet. Die größte Rauhigkeit beträgt also $15 \%$ des Rohrradius. Das Ergebnis ist aus Abb. 3 zu ersehen. Wie zu erwarten war, streuen hier die Punkte mehr als bei glatten Roh- 
ren. Sie liegen aber auch hier immer um denselben Mittelwert, und die sinngemäß durch die Punkte gelegte Gerade deckt sich mit der Geraden für glatte Rohre. Man erkennt daraus, daß die Rauhigkeit keinen Einfluß auf den Durchmesser des ausgezeichneten Zylindermantels hat.

Es sei noch erwähnt, daß L. A. O t t ${ }^{5}$ mit einer Gleichung, die sonst kaum die Gesetze der turbulenten Strömung erfüllt, Geschwindigkeitskurven aufstellt, die sich in einem ähnlichen Punkte schneiden.

\section{Neues Meßgerät}

Auf Grund der neuen Erkenntnis läßt sich ein sehr einfaches, aber trotzdem sehr genau arbeitendes Meßgerät entwickeln. Eine seiner Ausführungen besteht z. B. darin, daß man einen Meßring mit gleichem Innendurchmesser wie die Rohrleitung zwischen zwei Flansche derselben einbaut. In diesem Meßring ist ein Staurohr so angeordnet, daß es genau auf dem ausgezeichneten Zylindermantel den dynamischen Druck mißt, der als identisch mit dem mittleren dynamischen Druck erkannt wurde. Aus ihm läßt sich, wenn die Zustandsgrößen des Mediums bekannt sind, die mittlere Geschwindigkeit und damit auch die durchfließende Menge nach den bekannten Gleichungen

$$
\begin{gathered}
w_{m}=\sqrt{\frac{2 g}{\gamma} \Delta P} \quad[\mathrm{~m} / \mathrm{sec}] \\
G=F w_{m} \quad\left[\mathrm{~m}^{3} / \mathrm{sec}\right] \\
G^{\prime}=\gamma \cdot F \cdot w_{m} \quad[\mathrm{~kg} / \mathrm{sec}]
\end{gathered}
$$

bestimmen. Hierin bedeuten: $\Delta P\left[\mathrm{~kg} / \mathrm{m}^{2}\right]$ mittlerer dynamischer Druck, $\gamma\left[\mathrm{kg} / \mathrm{m}^{3}\right]$ spezif. Gewicht des Mediums, $g\left[\mathrm{~m} / \mathrm{sec}^{2}\right]$ Erdbeschleunigung, $F\left[\mathrm{~m}^{2}\right]$ Querschnitt der Rohrleitung, $G$ $\left[\mathrm{m}^{3} / \mathrm{sec}\right]$ bzw. $G^{\prime}[\mathrm{kg} / \mathrm{sec}]$ durchfließende Menge.

Einen Aufschluß über die Genauigkeit dieser Geräte geben uns die in Abb. 1 bis 3 dargestellten Meßergebnisse, nach denen in glatten Rohren bei der größten Streuung ein Fehler in der Geschwindigkeitsmessung und damit auch in der Mengenmessung von höchstens $0,7 \%$ auftreten würde. Selbst bei den größten Abweichungen dieser Werte im rauhen Rohr beträgt der Fehler höchstens 2,5\%. Bei gut ausgebildeter turbulenter Strömung ist also die Genauigkeit dieser Geräte bei glatten Rohren weit größer als bei den bisher bekannten Meßgeräten und der Fehler selbst bei rauhen Rohren etwa von der Größe der zugelassenen Fehler der Normgeräte. Der große Vorteil des neuen Gerätes besteht jedoch in der Einfachheit der Herstellung und dem geringen Druckverlust.

Sind Krümmer oder Absperrorgane nicht genügend weit von der Meßstelle entfernt, oder kann eine gut ausgebildete turbulente Strömung nicht gewährleistet werden, so könnte man auf dem ausgezeichneten Zylindermantel zwei oder mehrere Staurohre anbringen. Der Mittelwert aus den Ablesungen ergibt auch dann noch mit genügender Genauigkeit den mittleren dynamischen Druck der Strömung.

5 L. A. Ott, Wassermessung bei Wasserkraftanlagen, Wasserkraftjahrbuch 1924, S. 263. 